\section{Primary Gastric Plasmacytoma at an Early Stage: Unremarkable Effect of Eradication of Helicobacter pylori}

Key words: gastric plasmacytoma, eradication, Helicobacter pylori

Primary gastric plasmacytoma is a relatively rare disease, and many of the cases are not detected until the advanced stage. In nearly all cases, surgical treatment and/or chemotherapy or irradiation has been performed. However, the effectiveness of Helicobacter pylori $(\mathrm{Hp})$ eradication of the disease at the early stage, with invasion confined to the submucosal layer, has been recently reported $(1,2)$. Here, we report a rare case of early stage gastric plasmacytoma, with unremarkable effect of $\mathrm{Hp}$ eradication.

A 57-year-old man was examined by medical check, and an upper gastrointestinal series revealed a $2-\mathrm{cm}$ polypoid mass on the lesser curvature of the gastric corpus. He was referred to Sumitomo Hospital for further examination on June 12,2001 . He had no symptoms. His past and familial histories were unremarkable. On physical examination, neither an abdominal mass nor peripheral lymphadenopathy was detected. No abnormalities were found in laboratory examinations. Serum immunoglobulins, interleukin-6 and soluble interleukin-2 receptor levels were within the normal range. Neither M-protein in the serum nor Bence Jones protein in the urine was detected. Radiographs of the chest, abdomen, cranium, and skeleton revealed no abnormalities. Bone marrow biopsy was normal. There was no abnormal accumulation of radioisotopes on Ga-citrate or technetium. Abdominal ultrasound and computed tomography showed neither hepatosplenomegaly nor lymphadenopathy. Upper gastrointestinal endoscopy demonstrated a polypoid lesion with an irregular surface in the gastric corpus (Fig. 1A). Endoscopic ultrasonography (EUS) revealed a low echoic mass-like lesion under the first sonographic layer located in the polypoid lesion (Fig. 1B). Hp infection was confirmed by a positive rapid urease test, positive culture, histological examination, and detection of anti-Hp IgG in the serum.

Histological examination of biopsy samples taken from the gastric lesion revealed atypical plasma cells compactly infiltrated in the gastric mucosa (Fig. 1C). Some of these cells were binucleolar, and a few mitoses were seen. There was no proliferation of centrocyte-like cells or other inflammatory cells, and no lymph-epithelial lesions (Fig. 1D). Immunohistochemically, the plasma cells were positive for IgA (Fig. 1E) and lamda light chain (Fig. 1F), but they did not express kappa light chain, IgM or IgG. In addition, the cells were positive for leukocyte common antigen (CD45) and CD38, but negative for CD20, CD30 and CD3. From these results, we diagnosed this as a case of primary gastric plasmacytoma. After informed consent was obtained, Hp eradication was done by means of triple drug therapy consisting of lansoprazole $30 \mathrm{mg}$ twice daily, amoxicillin 750 $\mathrm{mg}$ twice daily, and clarithromycin $400 \mathrm{mg}$ twice daily for a week. Two months after completing this therapy, the eradication was confirmed by rapid urease test, histological examination, culture and a ${ }^{13} \mathrm{C}$-urea breath test. Upper gastrointestinal endoscopic and histological examinations were performed at 2, 4, 8 months after the eradication. At the last examination, the tumor looked unremarkably changed on endoscopic examination, the thickness of the low echoic lesion was unchanged by EUS, diffusely infiltrated atypical plasma cells were histologically found, and monotypic positivity for IgA-lamda of the cells was immunohistochemically demonstrated on examination of the biopsy samples.

On July 3, 2002 total gastrectomy with regional lymph node resection was performed. On microscopic examination of the resected specimen, clusters of plasma cells were observed in the propria mucosa of the polypoid lesion but these did not extend into the submucosa. Infiltration of inflammatory cells without atypism was observed in the surrounding of the tumor. Regional lymph nodes were free of atypical plasma cells.

Gastric plasmacytoma is a relatively rare disease and must be differentiated from mucosa-associated lymphoid tissue (MALT) lymphoma. In the present case, proliferation of centrocyte-like cells or a lymph-epithelial lesion was not found, and the tumor cells expressed CD38, but not CD20. From these findings this case can be differentiated from gastric MALT lymphoma histologically and immunochemically (3-5).

Gastric plasmacytoma has malignant potential, and surgical resection is generally performed. Recently, Kato et al (1) and Gonzalez et al (2) reported two cases of early stage gastric plasmacytoma, in which endoscopic regression of the tumors was observed at 3 months after Hp eradication, and proposed that $\mathrm{Hp}$ eradication may be considered as a firstline therapy for the disease at an early stage. In our case, however, in the 8-month observation after Hp eradication, the improvement of the tumor was unremarkable as demonstrated by endoscopic, EUS, and histological examinations although the plasmacytic proliferation was confined to the propria mucosa. In a review of English language literature, in most cases of gastric plasmacytoma it was not mentioned whether Hp was present or not. To our knowledge, no other report on the unremarkable effect of $\mathrm{Hp}$ eradication can be found. In addition, in the case of Kato et al (1), despite endoscopic regression, infiltration and monoclonality of the plasma cells still remained for 16 months after Hp eradication, and Gonzalez's case (2) had histological characteristics 


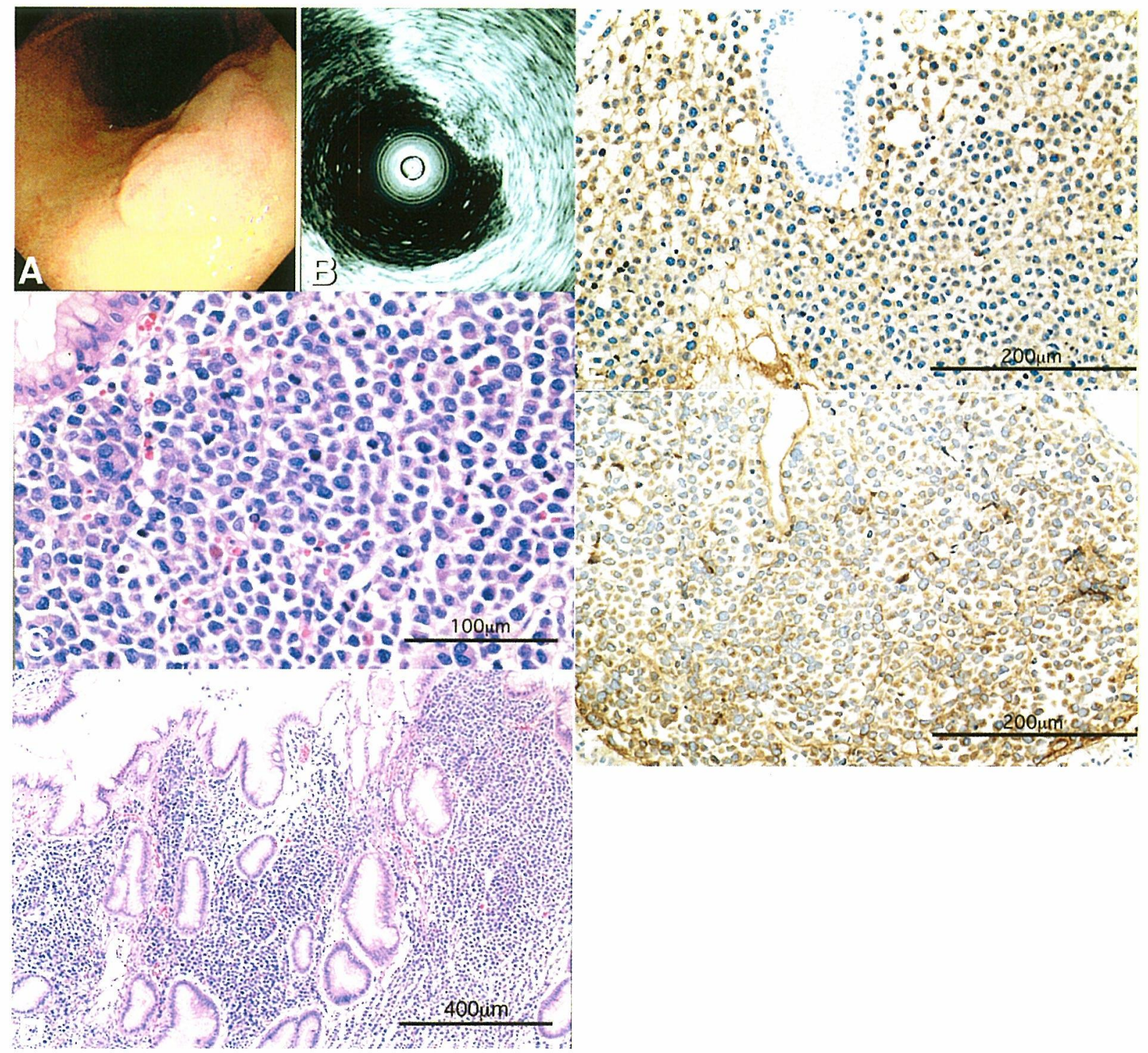

Figure 1. A: Upper gastrointestinal endoscopy demonstrated a polypoid lesion with an irregular surface on the lesser curvature of the gastric corpus. B: Endoscopic ultrasonography (20 MHz) showed a low echoic mass-like lesion under the first sonographic layer located in the polypoid lesion. Continuity of the second sonographic layer was observed. C: Histological examination of biopsy samples taken from the gastric polypoid lesion. Plasma cells compactly infiltrated in the gastric mucosa. Some of them were binucleolar, and a few mitoses were seen. (HE stain, $\times 100)$. Bar indicates 100 micrometer. D: Histological examination of biopsy samples. Lymph-epithelial lesion was not found. (HE stain, $\times 25)$. Bar indicates 400 micrometers. E: The atypical plasma cells were immunohistochemically stained with anti-IgA. (Immunostaining for IgA, ×62.5). Bar indicates 200 micrometers. F: The atypical plasma cells were immunohistochemically stained with anti-lamda light chain. (Immunostaining for lamda light chain, $\times 62.5)$. Bar indicates 200 micrometers.

of gastric MALT lymphoma, such as the presence of lymphepithelial lesion and accumulation of lymphoid cells, which were not commonly reported in cases of gastric plasmacytoma. Taking these reports and our case into account, although $\mathrm{Hp}$ eradication is non-invasive but low-risk, it remains to be clarified whether the eradication is effective for many cases of early stage gastric plasmacytoma.
Takeshi MinAmI, Shigeyuki NiSHIMURA*, Kohsuke ASAGOE**, Takahiro TSUJIMURA***,

Takahiro YAMAMOTO, Shinya KONDO, Yoshikazu OHMORI and Shuji KanAYAMA

The Department of Gastroenterology, *the Department of Surgery, **the Department of Internal Medicine and ***the Department of Pathology, Sumitomo Hospital, Osaka 
Received for publication October 9, 2002; Accepted for publication January 20, 2003

Reprint requests should be addressed to Dr. Takeshi Minami, the Department of Gastroenterology, Sumitomo Hospital, 5-3-20 Nakanoshima, Kitaku, Osaka 530-0005

\section{References}

1) Kato K, Sugitani M, Nagata $T$, et al. A case of gastric plasmacytoma associated with Helicobacter pylori infection: improvement of abdominal endoscopic and EUS findings after $H$. pylori eradication. Gastrointest Endosc 53: 352-355, 2001.
2) Gonzalez Cueto D, Bruno S, Bustos Fernandez LM, Narbaitz M. Gastric solitary plasmacytoma associated with + Helicobacter pylori infection. Acta Gastroenterol Latinoam 29: 119-123, 1999.

3) Ishido T, Mori N. Primary gastric plasmacytoma: A morphological and immunohistochemical study of five cases. Am J Gastroenterol 87: 875878, 1992.

4) Ishido T, Mori N, Kikuchi M, Nakamura K. Primary gastric malignant lymphoma. A morphological and immunohistochemical study of 38 cases. Acta Pathol Jpn 39: 229-234, 1989.

5) Harris NL, Jaffe ES, Stein H, et al. A revised European-American classification of lymphoid neoplasms: A proposal from the International Lymphoma Study Group. Blood 84: 1361-1392, 1994. 